\title{
Effectiveness of infliximab in refractory FDG PET-positive sarcoidosis
}

\author{
Adriane D.M. Vorselaars ${ }^{1,6}$, Heleen A. Crommelin ${ }^{1,2,6}$, Vera H.M. Deneer², \\ Bob Meek ${ }^{3}$, Anke M.E. Claessen ${ }^{3}$, Ruth G.M. Keijsers ${ }^{4}$, \\ Coline H.M. van Moorsel ${ }^{1,5}$ and Jan C. Grutters ${ }^{1,5}$
}

Affiliations: ${ }^{1}$ Interstitial Lung Diseases Centre of Excellence, Dept of Pulmonology, St Antonius Hospital, Nieuwegein, The Netherlands. ${ }^{2}$ Dept of Clinical Pharmacy, St Antonius Hospital, Nieuwegein, The Netherlands. ${ }^{3}$ Dept of Medical Microbiology and Immunology, St Antonius Hospital, Nieuwegein, The Netherlands. ${ }^{4}$ Dept of Nuclear Medicine, St Antonius Hospital, Nieuwegein, The Netherlands. ${ }^{5}$ Division of Heart and Lungs, University Medical Centre Utrecht, Utrecht, The Netherlands. ${ }^{6}$ Both authors contributed equally.

Correspondence: Jan C. Grutters, Interstitial Lung Diseases Centre of Excellence, Dept of Pulmonology, St Antonius Hospital, Koekoekslaan 1, 3435 CM Nieuwegein, The Netherlands.

E-mail: j.gruttersdantoniusziekenhuis.nl

ABSTRACT Inconclusive evidence for the efficacy of infliximab in sarcoidosis hinders the global use of this potentially beneficial drug. To study infliximab efficacy in a clinical setting, we performed a prospective open-label trial in patients refractory to conventional treatment.

Patients $(n=56)$ received eight infusions of $5 \mathrm{mg} \cdot \mathrm{kg}^{-1}$ infliximab. Pulmonary function, disease activity measured by ${ }^{18} \mathrm{~F}$-fluorodeoxyglucose (FDG) by positron emission tomography (PET) and quality of life were part of the clinical work-up. Infliximab levels were measured before every infusion.

After 26 weeks of infliximab treatment, mean improvement in forced vital capacity (FVC) was $6.6 \%$ predicted $(\mathrm{p}=0.0007)$, whereas in the 6 months before start of treatment, lung function decreased. Maximum standardised uptake value (SUVmax) of pulmonary parenchyma on ${ }^{18}$ F-FDG PET decreased by $3.93(\mathrm{p}<0.0001)$. High SUVmax of pulmonary parenchyma at baseline predicted FVC improvement $(\mathrm{R}=0.62, \mathrm{p}=0.0004)$. An overall beneficial response was seen in $79 \%$ of patients and a partial response was seen in $17 \%$ of patients. No correlation between infliximab trough level (mean $18.0 \mu \mathrm{g} \cdot \mathrm{mL}^{-1}$ ) and initial response was found.

In conclusion, infliximab causes significant improvement in FVC in refractory ${ }^{18}$ F-FDG PET positive sarcoidosis. Especially in pulmonary disease, high ${ }^{18}$ F-FDG PET SUVmax values at treatment initiation predict clinically relevant lung function improvement. These results suggest that inclusion of ${ }^{18}$ F-FDG PET is useful in therapeutic decision-making in complex sarcoidosis.

@ERSpublications

Infliximab is highly effective in refractory ${ }^{18} \mathrm{~F}$-FDG PET positive sarcoidosis patients http://ow.ly/JoxhL

Received: Sept 152014 | Accepted after revision: Feb 02 2015 | First published online: April 302015

Support statement: This study was supported by a research grant from the St Antonius Hospital innovation fund. Funding information for this article has been deposited with FundRef.

Conflict of interest: None declared.

This study is registered at www.trialregister.nl with identifier number NTR3895.

Copyright OERS 2015 


\section{Introduction}

Sarcoidosis is a systemic disease with a wide variety of symptoms and is histologically characterised by the formation of noncaseating granulomas [1]. Although the disease is often self-limiting, it can also follow a chronic course in a subgroup of patients $[2,3]$. Self-limiting disease does not necessitate treatment, but severe disease with organ failure or unacceptable loss of quality of life requires therapeutic intervention. When immunosuppressive treatment is indicated, corticosteroids remain the first-choice therapy $[4,5]$. Even though corticosteroids are generally effective, continued use is known to have severe side-effects, such as diabetes mellitus, osteoporosis or obesity [6]. Therefore, second-line therapy usually involves agents with steroid-sparing capacity, such as methotrexate or azathioprine [7-10].

Because some patients are refractory to these agents or develop considerable side-effects, biological agents targeted against tumour necrosis factor (TNF) have been introduced as a third-line treatment option [11]. The chimeric monoclonal anti-TNF drug infliximab (Remicade; Centocor, Inc., Malvern, PA, USA) has been extensively investigated and is widely used for treatment of immune-mediated inflammatory diseases such as Crohn's disease, rheumatoid arthritis and psoriasis [12-14], but a knowledge gap remains in the field of sarcoidosis treatment. Current recommendations are mostly derived from extrapolations from other chronic inflammatory diseases or based on experience and eminence-based medicine. In sarcoidosis, infliximab has shown positive results in retrospective series of several manifestations of sarcoidosis [15, 16], but the one large randomised controlled trial (RCT) investigating infliximab treatment in sarcoidosis only revealed a small improvement in pulmonary function and extrapulmonary symptoms after 24 weeks of treatment $[17,18]$. Critics doubt whether this small improvement of $2.5 \%$ in forced vital capacity (FVC) is clinically relevant $[19,20]$. Because this is the only prospective study on infliximab in sarcoidosis, more evidence is needed to determine efficacy and assess which patients will benefit most. Unfortunately, pharmaceutical companies are hesitant to further invest in the field of orphan diseases, especially when a drug has already been approved for other treatment indications [21]. Furthermore, as positive effects have been described, it is considered unethical to perform another RCT in this category of patients with severe disease and organ failure. In this unfortunate situation, in the absence of phase III RCT trials, the use of infliximab in refractory sarcoidosis is still not endorsed by healthcare insurance companies in many countries and remains off-label globally [22].

A key question remains: how to select the patients who will benefit most from this expensive therapy. Patients with high activity on ${ }^{18} \mathrm{~F}$-fluorodeoxyglucose (FDG) by positron emission tomography (PET) were often shown to relapse after infliximab therapy was discontinued [23], possibly suggesting a good initial response in those patients.

Besides patient selection, knowledge of the interindividual variance in response is derived from the use of infliximab in rheumatic diseases and gastroenterology. In these diseases it is known that formation of antibodies against infliximab can result in decreased levels of infliximab and diminished drug efficacy. Additionally, antibodies against infliximab can sometimes cause allergic reactions [12, 24-26]. It is not known whether formation of antibodies against infliximab and associated low trough levels play a role in treatment effect in the case of sarcoidosis.

The aim of this trial was to study for the first time the effect of infliximab in a prospective clinical setting, and to investigate whether sarcoidosis phenotype, inflammatory activity, infliximab trough levels or formation of antibodies against infliximab are related to the initial response rate after 26 weeks.

\section{Material and methods}

Study subjects

All sarcoidosis patients in whom infliximab therapy was initiated at St Antonius Hospital (Nieuwegein, The Netherlands), between January 2011 and April 2013, were invited to participate in this prospective, open-label cohort study. St Antonius Hospital Nieuwegein is a national tertiary referral centre for sarcoidosis. Only patients with severe sarcoidosis, unresponsive to first- and second-line treatment, or who have experienced severe side-effects from these agents (e.g. worsening diabetes, psychological deterioration or liver function disorders) were eligible for inclusion in the study. The diagnosis of sarcoidosis was made according to American Thoracic Society/European Respiratory Society criteria [1]. The treating physician judged disease severity at the moment of initiation based on loss of function (e.g. lung function or cardiac function), impaired quality of life and disease activity on ${ }^{18}$ F-FDG PET. Exclusion criteria were vaccination with live or bacterial vaccines within the previous 3 months, active or untreated latent tuberculosis, serious infections in the last 2 months, serious right ventricular heart failure, active hepatitis, history of allergic reactions to monoclonal antibodies or their fragments, opportunistic infections within the last 6 months, $\mathrm{HIV}$, transplantation, known malignancy, pregnancy or breastfeeding. 
The institutional review board of St Antonius Hospital Nieuwegein (registration number LTME/R-10.13A, acronym INFLIXIMAB) and ethics committee approved the study and patients gave written informed consent.

\section{Treatment}

Patients received infliximab intravenously following a standard protocol starting with $5 \mathrm{mg} \cdot \mathrm{kg}^{-1}$ bodyweight at weeks 0 and 2 and then every 4 weeks over a period of 6 months. Dosing of prednisone could be tapered according to the judgement of the treating physician.

\section{Functional response}

Organ function was assessed by functional evaluation of the index organ (e.g. for patients with pulmonary sarcoidosis we used FVC, forced expiratory volume in $1 \mathrm{~s}$ (FEV1) and diffusing capacity of the lung for carbon monoxide corrected for haemoglobin (DLCOC)). Small fibre neuropathy was tested using the small fibre neuropathy screening list and clinical judgement by the treating neurologist [27]. As the minimal important difference for change in FVC has not been elucidated in sarcoidosis, we also reported mean change in $\%$ predicted and percentage of patients with an increase of $\geqslant 5 \%$ pred and $\geqslant 10 \%$ pred [28].

\section{Inflammatory response}

Parameters in the inflammation category included the biomarkers soluble interleukin-2 receptor (sIL-2R), angiotensin-converting enzyme (ACE) and ${ }^{18} \mathrm{~F}$-FDG PET maximum standardised uptake value (SUVmax) of the pulmonary parenchyma and, if applicable, other index localisation of sarcoidosis. ${ }^{18}$ F-FDG PET imaging was performed using a Philips Gemini TF-64 combined PET/CT device (Philips Medical Systems, Eindhoven, The Netherlands). The SUVmax was calculated in the mediastinal/hilar region, in the lung parenchyma and in the target organ when appropriate. Regions of interest (ROIs) were drawn over the visually affected part of the organ to measure the SUVmax. ROI was drawn at the same lesion/area at baseline and follow-up scan. ROI drawing was performed using the automatic ROI drawing tool in the Hermes Diagnostics programme (Hermes Medical Solutions, Stockholm, Sweden). Blood glucose level was measured before injecting FDG in all patients. FDG was administered when the plasma glucose level was $<7 \mathrm{mmol} \cdot \mathrm{L}^{-1}$.

\section{Quality of life}

Finally, quality of life was measured using two questionnaires: a patient global assessment (PGA) score ranging from 0 (best imaginable health status) to 100 (worst imaginable health status) on a visual analogue scale and physical functioning on the short form (SF)-36 health survey. An improvement of 10 points was considered clinically relevant.

\section{Composite overall response}

In addition we reported response rate as a composite of three categories: organ function, inflammation and quality of life. Improvement in a category was scored only when one of the parameters improved significantly without deterioration of the others. For the functional response in pulmonary sarcoidosis we used an improvement in FVC of $\geqslant 5 \%$ pred. A decrease in biomarkers or SUVmax $>40 \%$ of baseline was considered a relevant improvement. Changes within the normal range of sIL-2R, ACE and SUVmax on ${ }^{18}$ F-FDG PET were not taken into account when gauging response. Change in SUVmax of the target organ was regarded as superior to a change in inflammatory biomarkers. Clinically relevant improvement in two or three categories was considered a good or excellent response, in one category as a partial response and in none of the categories as nonresponse.

\section{Infliximab trough levels and antibodies against infliximab}

Infliximab trough levels were measured using ELISA (Sanquin, The Netherlands) [29]. This ELISA only detects infliximab that is able to bind TNF. It does not detect immune complexes consisting of infliximab and TNF or infliximab bound to neutralising antibodies against infliximab. The presence of antibodies against infliximab was determined using radioimmunoassay [30].

\section{Analysis}

Changes in values before and after 6 months of treatment were compared using two-tailed paired t-tests. Pearson's correlation coefficients (R) between parameters were calculated using linear regression. Statistical analysis was performed using the SPSS for Windows (version 22.0; IBM, Armonk, NY, USA). Graphs were created using GraphPad Prism 5.0 (GraphPad Software, La Jolla, CA, USA). p $<0.05$ was considered significant. 


\section{Results}

Study subjects

Between January 2011 and April 2013, infliximab therapy was initiated in a total of 58 active refractory sarcoidosis patients, two of whom were not included in this study (fig. 1). 56 patients were included in the study, $64.3 \%$ of whom were male and $87.5 \%$ Caucasian (table 1). The most common treatment indication was pulmonary sarcoidosis (60.7\%); other common indications were cutaneous sarcoidosis and small fibre neuropathy. The vast majority of patients suffered from chronic sarcoidosis, with a mean disease duration at initiation of 6.8 years and the use of at least two immunosuppressant drugs prior to infliximab initiation in 92.9\% of patients. Furthermore, patients had signs of high disease activity, with a mean SUVmax of 6.6 on ${ }^{18} \mathrm{~F}$-FDG PET in the pulmonary parenchyma, a high sIL-2R of $8824 \mathrm{pg} \cdot \mathrm{mL}^{-1}$ (normal value $<3000 \mathrm{pg} \cdot \mathrm{mL}^{-1}$ ) and a high ACE of $89.7 \mathrm{U} \cdot \mathrm{L}^{-1}$ (normal value $<68 \mathrm{U} \cdot \mathrm{L}^{-1}$ ) at start of therapy. Baseline SUVmax on ${ }^{18} \mathrm{~F}-\mathrm{FDG}$ PET did not correlate with Scadding stages 0-III versus Scadding stage IV (6.3 versus 6.8, respectively; $\mathrm{p}=0.76)$.

\section{Organ function}

Of the total study population, 52 (93\%) patients had decreased pulmonary function (one or more pulmonary function test (PFT) parameters $<80 \%$ ). In patients with a pulmonary treatment indication, FVC increased by $6.64 \%$ pred $(\mathrm{p}=0.0007)$, FEV1 increased by $5.80 \%$ pred $(\mathrm{p}<0.0001)$ and DLCOc increased by $4.12 \%$ pred $(\mathrm{p}=0.001)$ after 6 months of infliximab treatment (table 2). An improvement of $\geqslant 5 \%$ pred FVC and FEV1 was seen in $71 \%$ and $64 \%$ of patients, respectively. In $46 \%$ of patients this increase exceeded $10 \%$ pred (fig. 2a).

Notably, even in patients with an extrapulmonary treatment indication, mean FVC and FEV1 increased significantly, by $3.88 \%$ pred $(\mathrm{p}=0.027)$ and $3.54 \%$ pred $(\mathrm{p}=0.034)$, respectively. Moreover, an increase of $\geqslant 5 \%$ pred was seen in $37 \%$ of these patients for each of these parameters (fig. $2 a$ ).

Prior to initiation of infliximab, stable or deteriorating pulmonary function was seen in the total cohort (fig. 2b). Using repeated measurement ANOVA, FVC after 26 weeks of treatment was found to be significantly higher than at initiation of treatment and than at 6 months before treatment $(\mathrm{p}<0.0001$ and $\mathrm{p}=0.007$, respectively). Baseline pulmonary function tests did not predict outcome (data not shown).

All four patients with cutaneous sarcoidosis had marked improvement or total resolution of skin lesions confirmed by photograph and clinical comparison. Patients with small fibre neuropathy had subjective improvement of symptoms.

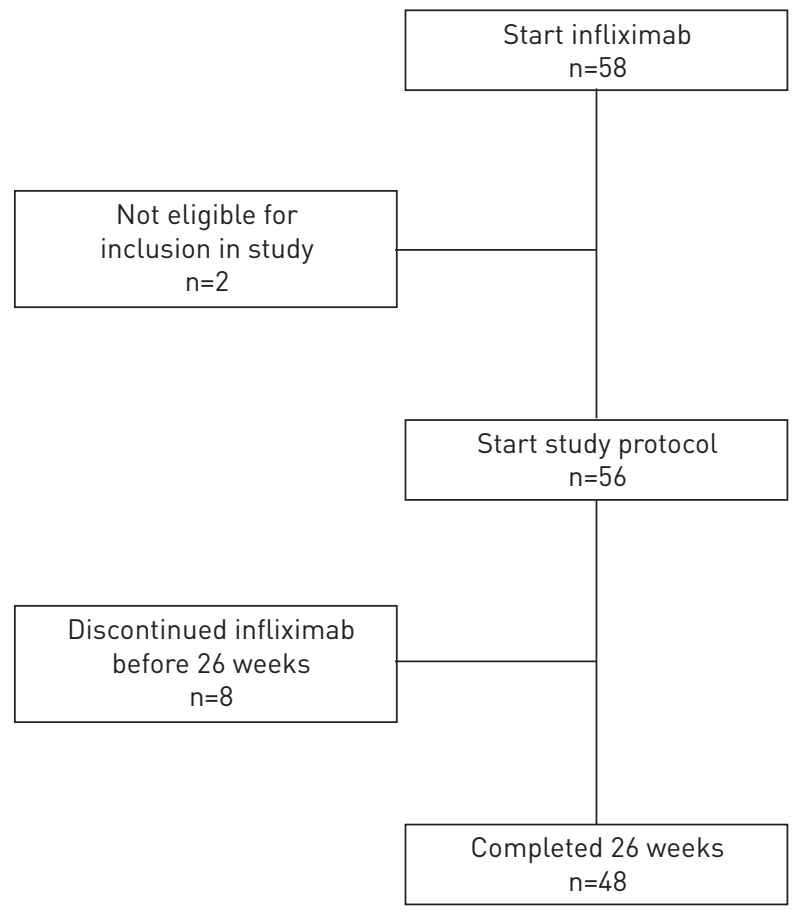

FIGURE 1 Flowchart of patient inclusion. Two patients were excluded from the study protocol, one due to incapability to give informed consent based on mental retardation and one because his cold agglutinin disease would not permit routine sampling at every visit. 
TABLE 1 Baseline characteristics of study subjects

\begin{tabular}{|c|c|}
\hline Subjects $n$ & 56 \\
\hline Male & $36(64.3)$ \\
\hline Caucasian & 49 (87.5) \\
\hline Age at initiation of infliximab therapy years & $48.7 \pm 10.1$ \\
\hline Disease duration at initiation of infliximab therapy years & $6.8 \pm 7.1$ \\
\hline \multicolumn{2}{|l|}{ Diagnosis of sarcoidosis } \\
\hline Biopsy & 52 (92.9) \\
\hline Bronchoalveolar lavage & $3(5.4)$ \\
\hline Clinical & $1(1.8)$ \\
\hline \multicolumn{2}{|l|}{ Smoking status } \\
\hline Never-smokers & $27(48.2)$ \\
\hline Current smokers & $4(7.1)$ \\
\hline Former smokers & $25(44.6)$ \\
\hline \multicolumn{2}{|l|}{ Scadding stage } \\
\hline 0 & $5(8.9)$ \\
\hline । & $6(10.7)$ \\
\hline II & $16(28.6)$ \\
\hline III & $14(25.0)$ \\
\hline IV & $15(26.8)$ \\
\hline \multicolumn{2}{|l|}{ Main treatment indication } \\
\hline Pulmonary & $34(60.7)$ \\
\hline Cardiac & $2(3.6)$ \\
\hline Small fibre neuropathy & 8 (14.3) \\
\hline Cutaneous & $4(7.2)$ \\
\hline Central nervous system & $3(5.4)$ \\
\hline Sinus & $1(1.8)$ \\
\hline Myositis & $1(1.8)$ \\
\hline Vocal cord paralysis & $1(1.8)$ \\
\hline Ossal & $1(1.8)$ \\
\hline Hypercalcaemia & $1(1.8)$ \\
\hline Tertiary referral & 52 (92.9) \\
\hline \multicolumn{2}{|l|}{ Medication use prior to initiation of infliximab } \\
\hline Corticosteroids & $54(96.4)$ \\
\hline Methotrexate & $51(91.1)$ \\
\hline Azathioprine & $6(10.7)$ \\
\hline Leflunomide & $1(1.8)$ \\
\hline Plaquenil & $8(14.3)$ \\
\hline Anti-TNF treatment & $13(23.2)$ \\
\hline None & $0(0)$ \\
\hline Use of $\geqslant 2$ different drugs prior to infliximab & $52(92.2)$ \\
\hline \multicolumn{2}{|l|}{ Concomitant medication } \\
\hline Corticosteroids & $24(42.9)$ \\
\hline Methotrexate & $46(82.1)$ \\
\hline Azathioprine & $4(7.1)$ \\
\hline Leflunomide & $1(1.8)$ \\
\hline None & $0(0)$ \\
\hline \multicolumn{2}{|l|}{ Pulmonary function parameters } \\
\hline FVC L (\% pred) & $3.32(78.8)$ \\
\hline FEV 1 L (\% pred $)$ & $2.30(66.8)$ \\
\hline DLcoc L (\% pred) & $6.01(59.8)$ \\
\hline 6MWD m (\% pred) & $460.4(62.2)$ \\
\hline \multicolumn{2}{|l|}{ Measurements of disease activity and severity } \\
\hline SUVmax lung parenchyma & $6.6 \pm 5.3$ \\
\hline SUVmax mediastinum & $5.7 \pm 3.2$ \\
\hline SUVmax total (including index localisation) & $9.0 \pm 5.2$ \\
\hline$A C E U \cdot L^{-1}$ & $89.73 \pm 49.7$ \\
\hline ACE Z-score & $4.26 \pm 4.8$ \\
\hline $\mathrm{sIL}-2 \mathrm{R} \mathrm{pg} \cdot \mathrm{mL}^{-1}$ & $8824 \pm 8503$ \\
\hline
\end{tabular}

Data are presented as $\mathrm{n}(\%)$ or mean \pm SD, unless otherwise stated. TNF: tumour necrosis factor; FVC: forced vital capacity; \% pred: \% predicted; FEV1: forced expiratory volume in $1 \mathrm{~s}$; DLCOc: diffusing capacity for carbon monoxide corrected for haemoglobin; 6MWD: 6-min walking distance; SUVmax: maximum standardised uptake value on ${ }^{18} \mathrm{~F}$-fluorodeoxyglucose by positron emission tomography; ACE: angiotensin-converting enzyme; sIL-2R: soluble interleukin-2 receptor. 
TABLE 2 Baseline disease parameters and change after 26 weeks of infliximab treatment in patients with pulmonary treatment indication

Baseline Change after infliximab treatment

\begin{tabular}{lcc}
\hline Pulmonary function parameters & & \\
FVC \% pred & 73.6 & +6.6 \\
FEV $\%$ pred & 55.8 & +5.8 \\
DLCOC \% pred & 56.6 & +4.1 \\
6MWD \% pred & 61.0 & +4.2 \\
Disease activity and severity measurements & & \\
SUVmax lung parenchyma & $9.0 \pm 5.0$ & $-5.3 \pm 5.6$ \\
SUVmax mediastinum & $5.9 \pm 3.3$ & $-2.7 \pm 3.8$ \\
SUVmax index localisation & $9.8 \pm 5.3$ & $-5.5 \pm 5.6$ \\
ACE U.L $\mathrm{L}^{-1}$ & $86.2 \pm 46.3$ & $-21.8 \pm 43.3$ \\
ACE Z score & $3.7 \pm 3.9$ & $-1.78 \pm 3.33$ \\
SIL-2R pg. $\mathrm{mL}^{-1}$ & $7631 \pm 4259$ & $-3955 \pm 3883$
\end{tabular}

$\mathrm{n}=28$. Data are presented as mean \pm SD, unless otherwise stated. FVC: forced vital capacity; $\%$ pred: \% predicted; FEV1: forced expiratory volume in $1 \mathrm{~s}$; DLCOc: diffusing capacity for carbon monoxide corrected for haemoglobin; 6MWD: 6-min walking distance; SUVmax: maximum standardised uptake value on ${ }^{18} \mathrm{~F}$-fluorodeoxyglucose by positron emission tomography; ACE: angiotensin-converting enzyme; sIL-2R: soluble interleukin-2 receptor.

\section{Inflammatory activity}

The number of patients in whom serum sIL-2R exceeded the upper limit of normal $\left(3000 \mathrm{pg} \cdot \mathrm{mL}^{-1}\right)$ was 47 (84\%). Due to use of ACE inhibitors, data on ACE levels were only usable in 49 patients, 30 (61\%) of whom had levels $>68 \mathrm{U} \cdot \mathrm{L}^{-1}$. When measuring disease activity by means of ${ }^{18} \mathrm{~F}$-FDG PET scan before and after 26 weeks of treatment we found a decrease in SUVmax of mediastinum and lung parenchyma of 2.97 $(p<0.0001)$ and $3.93(p<0.0001)$, respectively. Moreover, the SUVmax of lungs and index localisation (e.g. heart) decreased significantly by $5.76(\mathrm{p}<0.0001)$ (fig. 3 ).

Both serum markers ACE and sIL-2R decreased significantly by $28.2 \mathrm{U} \cdot \mathrm{L}^{-1} \quad(\mathrm{p}=0.0003)$ and 4269.4 pg. $\mathrm{mL}^{-1}(\mathrm{p}<0.0001)$, respectively. Interestingly, ACE was higher in patients with an extrapulmonary treatment indication compared to patients with a pulmonary treatment indication $\left(97.8\right.$ and $86.2 \mathrm{U} \cdot \mathrm{L}^{-1}$, respectively).

Furthermore, we found significant correlations between the change in pulmonary function and level of disease activity, indicating that pulmonary function improved for the majority of patients with the highest disease activity. In patients with a pulmonary treatment indication, improvement in FVC following treatment correlated best with SUVmax of the pulmonary parenchyma before treatment initiation, having a correlation coefficient of $0.62(\mathrm{p}=0.0004)$ (fig. 4). Linear regression analysis, including the parameters
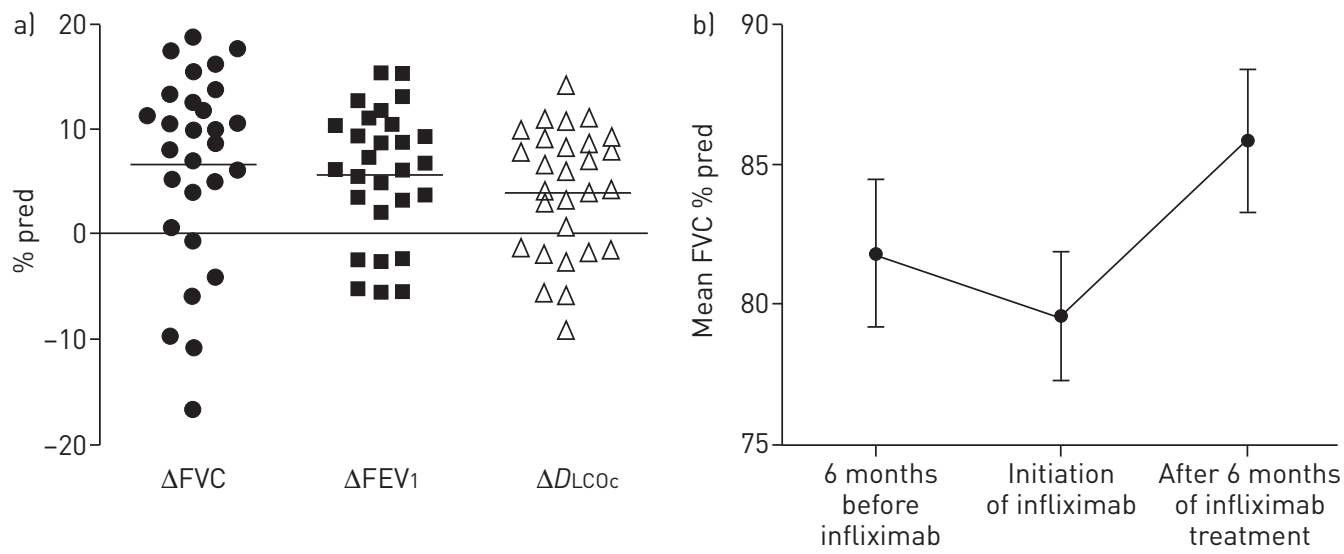

FIGURE 2 Pulmonary response in sarcoidosis. a) Change in pulmonary function after 26 weeks of infliximab therapy in patients with a pulmonary treatment indication (change in \% predicted); b) mean forced vital capacity (FVC) 6 months before initiation and during 26 weeks of infliximab therapy in the total cohort (\% pred). Bars represent SEM. FEV 1 : forced expiratory volume in $1 \mathrm{~s}$; DLCOc: diffusing capacity of the lung for carbon monoxide corrected for haemoglobin. 

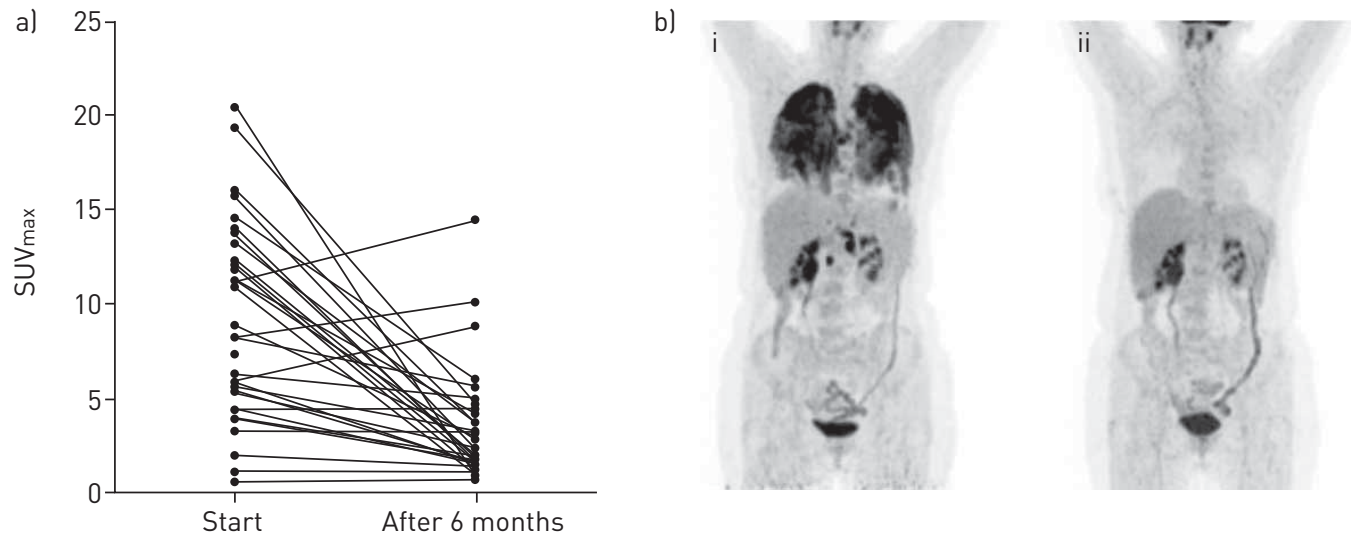

FIGURE $3{ }^{18}$ F-fluorodeoxyglucose (FDG) by positron emission tomography (PET) activity during treatment. a) Maximum standardised uptake value (SUVmax) on ${ }^{18} \mathrm{~F}$-FDG PET of the target organ at initiation and after 26 weeks of infliximab treatment. b) Example of ${ }^{18}$ F-FDG PET in a patient with pulmonary sarcoidosis i) before and ii) after 26 weeks of infliximab treatment.

SUVmax of the parenchyma and FVC at treatment initiation, predicted that FVC would improve by $1.1 \%$ per unit SUVmax of the parenchyma at start of therapy. Consequently, a SUVmax of 10 in the parenchyma at initiation predicts an FVC increase of $11 \%$ pred. Baseline sIL-2R correlated with improvement in DLCOC ( $\mathrm{R} 0.50, \mathrm{p}=0.007$ ), while ACE at baseline correlated with improvement in FEV1 in patients with an extrapulmonary treatment indication in particular $(\mathrm{R}$ 0.51, $\mathrm{p}=0.04)$. When comparing patients with deterioration of lung function measured by FVC (fig. 2a) to the other patients, SUVmax of the pulmonary parenchyma and sIL-2R at baseline were found to be significantly different. No significant correlation with ACE, PFTs, age, sex, Scadding stage, ethnicity or disease duration was found.

Prednisone was used concurrently in 19 patients at the start of infliximab therapy. The mean daily dose decreased by $8.8 \mathrm{mg}$ after 26 weeks of therapy $(\mathrm{p}=0.001)$. The dose of concomitant immunosuppressive drugs was increased in none of the patients.

\section{Quality of life}

Mean PGA score on a visual analogue scale at treatment initiation was 61.0 out of 100 (being worst imaginary health status) and showed a clinically significant decrease of -14.6 after 26 weeks of treatment $(\mathrm{p}<0.0001)$. The mean physical functioning score on the SF-36 was 40.6 out of 100 and increased by 8.2 $(\mathrm{p}=0.009)$ after 26 weeks of treatment.

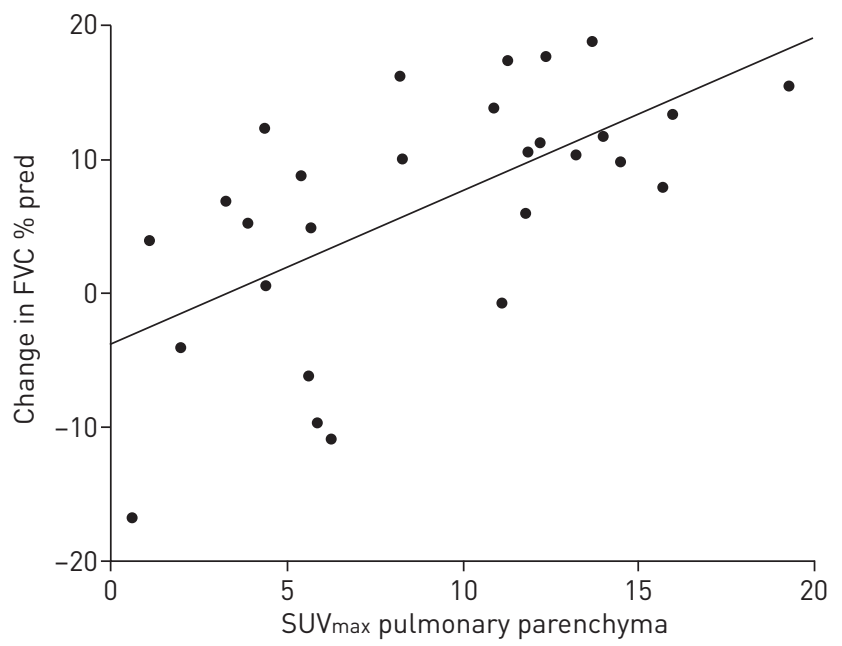

FIGURE $4{ }^{18} \mathrm{~F}$-fluorodeoxyglucose (FDG) by positron emission tomography (PET) activity and improvement in pulmonary function. Correlation between high activity of pulmonary parenchyma on ${ }^{18} \mathrm{~F}$-FDG PET (maximum standardised uptake value $\left(\mathrm{SUV}_{\max }\right)$ ) at baseline and improvement in forced vital capacity (FVC) in patients with a pulmonary treatment indication $(\mathrm{R}=0.62, \mathrm{p}=0.0004)$ 


\section{Composite overall response rate}

When evaluating response after 26 weeks of treatment as a composite of three categories (organ function, inflammatory activity and quality of life) we found a very high response rate (fig. 5). An excellent or good response was seen in $79 \%$ of patients, indicating clinically relevant improvement in at least two out of three categories. $17 \%$ of patients had a partial response, indicating improvement in one category. $4 \%$ of patients did not respond. When dividing response into the three categories, we found that $69 \%$ responded on the functional category, $79 \%$ on the inflammation category and $67 \%$ on the quality-of-life category. Patient characteristics, such as age, race, sex and disease duration did not predict overall response.

\section{Infliximab trough levels and antibodies against infliximab}

Infliximab trough levels showed high interindividual variation, but intraindividual variation was low throughout the 26 weeks of treatment. Trough levels were high: the mean trough level was $18.0 \mu \mathrm{g} \cdot \mathrm{mL}^{-1}$. No significant correlation between trough level and response was found. Patients with excellent or good response had a mean trough level of $18.5 \mu \mathrm{g} \cdot \mathrm{mL}^{-1}$, partial responders a mean trough level of $17.4 \mu \mathrm{g} \cdot \mathrm{mL}^{-1}$ and nonresponders a mean trough level of $27.5 \mu \mathrm{g} \cdot \mathrm{mL}^{-1}$.

Three patients showed continuously undetectable trough levels of infliximab. Two of these patients had an allergic reaction within 26 weeks of treatment. The other patient developed an allergic reaction after 1 year of treatment. Corresponding with low levels of infliximab, high levels of antibodies against infliximab were present in all three patients. All of these patients received concomitant immunosuppressive therapy during infliximab treatment: one patient received prednisone $20 \mathrm{mg} \cdot \mathrm{day}^{-1}$, one patient methotrexate $7.5 \mathrm{mg} \cdot \mathrm{week}^{-1}$ and one patient was on prednisone $10 \mathrm{mg} \cdot \mathrm{day}^{-1}$ and methotrexate $7.5 \mathrm{mg} \cdot \mathrm{week}^{-1}$.

\section{Side-effects and discontinuation of therapy}

Severe side-effects were pneumonia, requiring hospitalisation and discontinuation of therapy in three patients, one of whom was hospitalised in the intensive care unit after two infusions and one other who was hospitalised after three infusions and eventually died of respiratory failure. One patient was hospitalised with severe progressive disease after three infusions. Therapy was then discontinued and the patient died several months later at home of respiratory failure. Another patient, known to have peritoneal dialysis, initially responded well, but developed peritonitis, requiring discontinuation of treatment. In another patient, therapy was discontinued due to severe gastrointestinal complaints.

a)

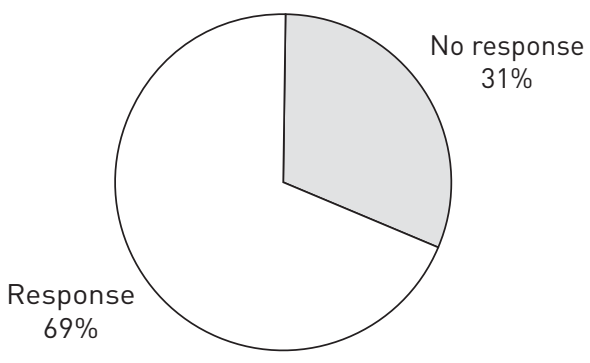

c)

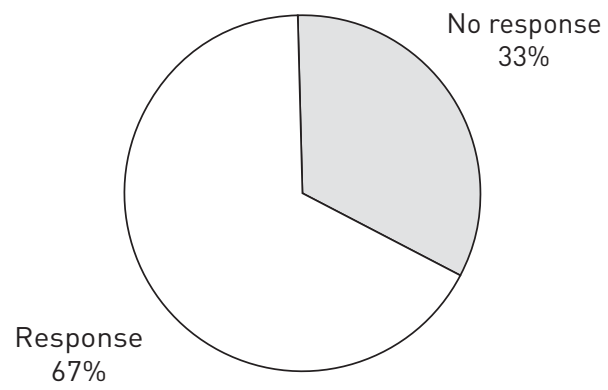

b)

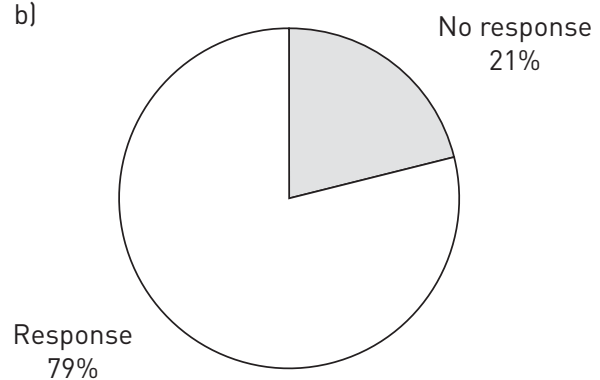

d)

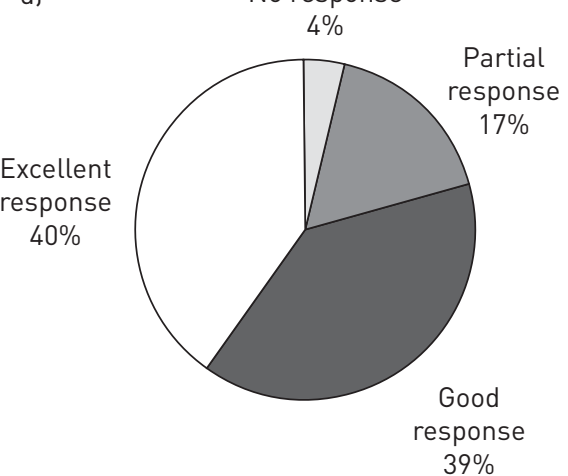

FIGURE 5 Response after 26 weeks of infliximab therapy. Response was measured in a) organ function, b) inflammation and c) quality of life; d) composite overall response. Excellent responders showed marked improvement in all three categories; good responders in two categories; and partial responders in one category. Nonresponders showed no marked improvement in any of the three categories. 
Allergic reactions along with antibody formation occurred in two patients within 26 weeks of treatment, one of whom discontinued infliximab treatment within 26 weeks. Both patients eventually successfully switched to adalimumab.

One patient did not want to continue infliximab therapy for undisclosed reasons.

Five patients had mild infections of the upper or lower respiratory system that did not require hospitalisation. Other side-effects were mild, such as headache $(n=2)$, dizziness $(n=1)$, oedema $(n=3)$ and joint pain $(n=2)$. The majority of patients had no side-effects $(n=34)$.

\section{Discussion}

In this prospective open-label trial of infliximab in active sarcoidosis patients refractory to conventional treatment, and including ${ }^{18} \mathrm{~F}$-FDG PET in the clinical work-up, we found a mean improvement of $6.6 \%$ pred in FVC and a very high overall response rate.

The only large RCT performed in this field only showed a small improvement of $2.5 \%$ pred in FVC, and no treatment benefit on other major secondary clinical end-points [17]. Importantly, only patients with stable disease were eligible for participation in the RCT. In contrast, the high response rate in our study might be attributable to the high disease activity measured by ${ }^{18}$ F-FDG PET in this cohort. This could be explained by the fact that infliximab, being an anti-inflammatory drug, probably finds more of its target in patients with higher inflammatory activity compared with those with lower or no sign of inflammatory activity on ${ }^{18}$ F-FDG PET.

Besides high activity on ${ }^{18}$ F-FDG PET, patients included in our study also had high serum levels of the disease activity markers ACE and sIL-2R. At initiation of infliximab therapy, mean levels of ACE and sIL-2R were $89.7 \mathrm{U} \cdot \mathrm{L}^{-1}$ and $8824 \mathrm{pg} \cdot \mathrm{mL}^{-1}$, respectively (with upper limit of normal for reference values $68 \mathrm{U} \cdot \mathrm{L}^{-1}$ and $3000 \mathrm{pg} \cdot \mathrm{mL}^{-1}$, respectively). These values are clearly higher than ACE at initiation of the large RCT of $47.4 \mathrm{U} \cdot \mathrm{L}^{-1}[18]$, or described in a recent retrospective cohort of $20.7 \mathrm{U} \cdot \mathrm{L}^{-1}$ (upper limit of normal $25 \mathrm{U} \cdot \mathrm{L}^{-1}$ in this study) [31]. In the latter study, mean sIL-2R at start of therapy was $3073 \mathrm{pg} \cdot \mathrm{mL}^{-1}$. In our study, the serum biomarkers ACE and sIL-2R were also found to decrease dramatically after 26 weeks of treatment and correlated with improvement in pulmonary function. Although the findings in our study might suggest that they could serve as less expensive surrogates for ${ }^{18}$ F-FDG PET, the value of these markers in sarcoidosis is still under debate [32]. Interpretation based on one measurement is more difficult due to high interpatient variability. Moreover, ACE and sIL-2R are less sensitive for detecting activity, and, most importantly, reflect systemic activity, whereas ${ }^{18}$ F-FDG PET can reveal specific organ involvement such as cardiac sarcoidosis $[33,34]$. In our view, ACE and sIL-2R are especially valuable for follow-up in individual patients.

Another explanation for the high improvement in FVC and high response rate found in our study compared to the only large RCT could be the difference in interval between infusions (4 versus 6 weeks). Infliximab levels were higher in our study than in the RCT (18.0 versus $\left.7.5 \mu \mathrm{g} \cdot \mathrm{mL}^{-1}\right)$ and also higher than those reported in other immune-mediated inflammatory diseases [35-37]. Consequently, it will be of interest to study whether a lower dose would achieve the same results in patients with active disease. The only RCT of infliximab in sarcoidosis did not show a difference between the groups treated with 3 and $5 \mathrm{mg} \cdot \mathrm{kg}^{-1}$ [17]; however, overall improvement was much lower in this RCT than in our study. A different dosing regimen could possibly further minimise toxicity and, moreover, could serve to reduce the unfortunately considerable costs of treatment with biologicals such as infliximab. Low trough levels were observed in just three patients during the first 26 weeks of treatment. It remains possible that low trough levels due to presence of antibodies against infliximab will occur more frequently after long-term treatment. Furthermore, concurrent use of methotrexate may have contributed to prevention of antibodies against infliximab formation in some patients, even though two out of three patients with antibodies against infliximab also received methotrexate.

In our clinic it is standard procedure to screen patients awaiting infliximab therapy for disease activity by measuring serum activity markers ACE and sIL-2R and performing ${ }^{18} \mathrm{~F}$-FDG PET. Though ${ }^{18} \mathrm{~F}-\mathrm{FDG}$ PET may be considered an expensive diagnostic tool, the value of being able to identify these patients with severe active sarcoidosis outweighs the costs, as treatment with the biological drug infliximab is over 10 times more expensive than performing ${ }^{18} \mathrm{~F}$-FDG PET. The use of ${ }^{18} \mathrm{~F}$-FDG PET is a valuable tool in identifying those patients for whom treatment with infliximab is expected to have beneficial effect.

A limitation of this study is the absence of a control group. Because of the large number of infliximab-treated patients described in case reports and case series, anti-TNF agents are incorporated into reviews guiding treatment of sarcoidosis $[6,19,28,38]$. Therefore, it is regarded unethical to perform an RCT whereby infliximab treatment is withheld from patients who are appointed to the placebo arm. To compare treatment results with conventional therapy, data from the time period prior to initiation of 
infliximab treatment have been used in data analysis as the best alternative to a placebo-controlled trial in the evaluation of treatment for rare diseases [39].

Previous studies have focused mainly on response of functional parameters rather than parameters regarding inflammatory activity or quality of life $[17,31]$. Reduction of inflammation, by infliximab, can be of clinical importance in possibly preventing future organ damage. Quality of life is an increasingly important, but under-reported outcome measure in sarcoidosis [40], which we therefore did include in the composite score. The combination of function, inflammation and quality of life (on a visual analogue scale) resembles the three categories also used in rheumatology in the disease activity score-28 [41, 42]. A limitation of our composite overall response score is that deterioration in one category, when another category is improving, is not taken into account. Therefore, this composite overall response should be interpreted with care and the response to the individual categories depicted in fig. $5 \mathrm{a}-\mathrm{c}$ should be regarded as leading. Furthermore, it has not yet been validated and future studies should reveal its value in clinical research.

Another possible limitation of the study is the high activity on ${ }^{18} \mathrm{~F}$-FDG PET and biomarkers in most patients in the study. Hereby we were able to show better results compared to the large RCT [17]. However, the observed correlation between ${ }^{18} \mathrm{~F}-\mathrm{FDG}$ PET and pulmonary improvement could hypothetically have been even stronger when patients without disease activity would have been included.

The pharmaceutical industry has shown no interest in obtaining registration of approval by the US Food and Drug Administration and European Medicines Agency for infliximab in sarcoidosis. This leaves physicians unable to prescribe the drug, unless this is done off-label based on evidence for efficacy mainly from observational data, with the additional consequence of low pharmacovigilance [21]. Furthermore, global financial endorsement by health insurance companies is unlikely to be granted without substantial evidence of effect. Our selection criteria and findings may convince health insurance companies to endorse infliximab therapy as treatment option for sarcoidosis. As trials investigating newer biologicals in sarcoidosis have been unsuccessful [43], infliximab remains the best option for the group of patients with severe refractory sarcoidosis.

In conclusion, infliximab therapy is very effective in selected patients with refractory disease and evidence of persistent disease activity. Patient selection for this indication should therefore ideally be based on both disease severity and inflammatory activity on ${ }^{18} \mathrm{~F}$-FDG PET. In addition, we have found that with the current fixed dosing regimen, levels of infliximab are high, suggesting room for dose reduction and associated cost reduction, e.g. by a flexible dosing regimen based on infliximab levels.

\section{Acknowledgements}

The authors would like to thank Pieter Zanen (University Medical Centre Utrecht, Utrecht, The Netherlands) for his excellent statistical advice.

\section{References}

1 Statement on sarcoidosis. Joint Statement of the American Thoracic Society (ATS), the European Respiratory Society (ERS) and the World Association of Sarcoidosis and Other Granulomatous Disorders (WASOG) adopted by the ATS Board of Directors and by the ERS Executive Committee, February 1999. Am J Respir Crit Care Med 1999; 160: 736-755.

2 Iannuzzi MC, Fontana JR. Sarcoidosis: clinical presentation, immunopathogenesis, and therapeutics. JAMA 2011; 305: 391-399.

3 Judson MA, Baughman RP, Thompson BW, et al. Two year prognosis of sarcoidosis: the ACCESS experience. Sarcoidosis Vasc Diffuse Lung Dis 2003; 20: 204-211.

4 Grutters JC, van den Bosch JM. Corticosteroid treatment in sarcoidosis. Eur Respir J 2006; 28: 627-636.

5 Paramothayan NS, Lasserson TJ, Jones PW. Corticosteroids for pulmonary sarcoidosis. Cochrane Database Syst Rev 2005; CD001114.

6 Vorselaars AD, van Moorsel CH, Deneer VH, et al. Current therapy in sarcoidosis, the role of existing drugs and future medicine. Inflamm Allergy Drug Targets 2013; 12: 369-377.

7 Baughman RP, Winget DB, Lower EE. Methotrexate is steroid sparing in acute sarcoidosis: results of a double blind, randomized trial. Sarcoidosis Vasc Diffuse Lung Dis 2000; 17: 60-66.

8 Lower EE, Baughman RP. Prolonged use of methotrexate for sarcoidosis. Arch Intern Med 1995; 155: 846-851.

9 Cremers JP, Drent M, Bast A, et al. Multinational evidence-based World Association of Sarcoidosis and Other Granulomatous Disorders recommendations for the use of methotrexate in sarcoidosis: integrating systematic literature research and expert opinion of sarcoidologists worldwide. Curr Opin Pulm Med 2013; 19: 545-561.

10 Vorselaars AD, Wuyts WA, Vorselaars VM, et al. Methotrexate vs azathioprine in second-line therapy of sarcoidosis. Chest 2013; 144: 805-812.

11 Drent M, Cremers JP, Jansen TL, et al. Practical eminence and experience-based recommendations for use of TNF- $\alpha$ inhibitors in sarcoidosis. Sarcoidosis Vasc Diffuse Lung Dis 2014; 31: 91-107.

12 Maini RN, Breedveld FC, Kalden JR, et al. Therapeutic efficacy of multiple intravenous infusions of anti-tumor necrosis factor alpha monoclonal antibody combined with low-dose weekly methotrexate in rheumatoid arthritis. Arthritis Rheum 1998; 41: 1552-1563.

13 Chaudhari U, Romano P, Mulcahy LD, et al. Efficacy and safety of infliximab monotherapy for plaque-type psoriasis: a randomised trial. Lancet 2001; 357: 1842-1847. 
14 D'haens G, Van Deventer S, Van Hogezand R, et al. Endoscopic and histological healing with infliximab anti-tumor necrosis factor antibodies in Crohn's disease: a European multicenter trial. Gastroenterology 1999; 116: 1029-1034.

15 van Rijswijk HN, Vorselaars AD, Ruven HJ, et al. Changes in disease activity, lung function and quality of life in patients with refractory sarcoidosis after anti-TNF treatment. Expert Opin Orphan Drugs 2013; 1: 437-443.

16 Hostettler KE, Studler U, Tamm M, et al. Long-term treatment with infliximab in patients with sarcoidosis. Respiration 2012; 83: 218-224.

17 Baughman RP, Drent M, Kavuru M, et al. Infliximab therapy in patients with chronic sarcoidosis and pulmonary involvement. Am J Respir Crit Care Med 2006; 174: 795-802.

18 Judson MA, Baughman RP, Costabel U, et al. Efficacy of infliximab in extrapulmonary sarcoidosis: results from a randomised trial. Eur Respir J 2008; 31: 1189-1196.

19 Valeyre D, Prasse A, Nunes H, et al. Sarcoidosis. Lancet 2014; 383: 1155-1167.

20 Maneiro JR, Salgado E, Gomez-Reino JJ, et al. Efficacy and safety of TNF antagonists in sarcoidosis: data from the Spanish registry of biologics BIOBADASER and a systematic review. Semin Arthritis Rheum 2012; 42: 89-103.

21 Vaz Carneiro A, Costa J. A prescrição fora das indicações aprovadas (off-label): prática e problemas [Off-label prescription: practice and problems.] Rev Port Cardiol 2013; 32: 681-686.

22 Zimmermann A, Dubaniewicz A, Slominski JM. Pharmacotherapy for sarcoidosis: an example of an off-label procedure. Adv Exp Med Biol 2013; 755: 251-256.

23 Vorselaars AD, Verwoerd A, van Moorsel $\mathrm{CH}$, et al. Prediction of relapse after discontinuation of infliximab therapy in severe sarcoidosis. Eur Respir J 2014; 43: 602-609.

24 Garcês S, Demengeot J, Benito-Garcia E. The immunogenicity of anti-TNF therapy in immune-mediated inflammatory diseases: a systematic review of the literature with a meta-analysis. Ann Rheum Dis 2013; 72: 1947-1955.

25 Steenholdt C, Svenson M, Bendtzen K, et al. Severe infusion reactions to infliximab: aetiology, immunogenicity and risk factors in patients with inflammatory bowel disease. Aliment Pharmacol Ther 2011; 34: 51-58.

26 Wang W, Wang EQ, Balthasar JP. Monoclonal antibody pharmacokinetics and pharmacodynamics. Clin Pharmacol Ther 2008; 84: 548-558.

27 Hoitsma E, De Vries J, Drent M. The small fiber neuropathy screening list: construction and cross-validation in sarcoidosis. Respir Med 2011; 105: 95-100.

28 Baughman RP, Nunes H, Sweiss NJ, et al. Established and experimental medical therapy of pulmonary sarcoidosis. Eur Respir J 2013; 41: 1424-1438.

29 Wolbink GJ, Voskuyl AE, Lems WF, et al. Relationship between serum trough infliximab levels, pretreatment C reactive protein levels, and clinical response to infliximab treatment in patients with rheumatoid arthritis. Ann Rheum Dis 2005; 64: 704-707.

30 Wolbink GJ, Vis M, Lems W, et al. Development of antiinfliximab antibodies and relationship to clinical response in patients with rheumatoid arthritis. Arthritis Rheum 2006; 54: 711-715.

31 Wijnen PA, Cremers JP, Nelemans PJ, et al. Association of the TNF- $\alpha$ G-308A polymorphism with TNF-inhibitor response in sarcoidosis. Eur Respir J 2014; 43: 1730-1739.

32 Keir G, Wells AU. Assessing pulmonary disease and response to therapy: which test? Semin Respir Crit Care Med 2010; 31: 409-418.

33 Adams H, Keijsers RG, Korenromp IH, et al. FDG PET for gauging of sarcoid disease activity. Semin Respir Crit Care Med 2014; 35: 352-361.

34 Keijsers RG, Verzijlbergen FJ, Oyen WJ, et al. ${ }^{18}$ F-FDG PET, genotype-corrected ACE and sIL-2R in newly diagnosed sarcoidosis. Eur J Nucl Med Mol Imaging 2009; 36: 1131-1137.

35 Bortlik M, Duricova D, Malickova K, et al. Infliximab trough levels may predict sustained response to infliximab in patients with Crohn's disease. J Crohns Colitis 2013; 7: 736-743.

36 van der Maas A, van den Bemt BJ, Wolbink G, et al. Low infliximab serum trough levels and anti-infliximab antibodies are prevalent in rheumatoid arthritis patients treated with infliximab in daily clinical practice: results of an observational cohort study. BMC Musculoskelet Disord 2012; 13: 184.

37 Takahashi H, Tsuji H, Ishida-Yamamoto A, et al. Plasma trough levels of adalimumab and infliximab in terms of clinical efficacy during the treatment of psoriasis. J Dermatol 2013; 40: 39-42.

38 Vorselaars AD, Cremers JP, Grutters JC, et al. Cytotoxic agents in sarcoidosis: which one should we choose? Curr Opin Pulm Med 2014; 20: 479-487.

39 Korn EL, McShane LM, Freidlin B. Statistical challenges in the evaluation of treatments for small patient populations. Sci Transl Med 2013; 5: 178sr3.

40 Korenromp IH, van de Laar MA. Health-related quality of life in sarcoidosis. Curr Opin Pulm Med 2014; 20: 503-507.

41 Eng G, Stoltenberg MB, Szkudlarek M, et al. Efficacy of treatment intensification with adalimumab, etanercept and infliximab in rheumatoid arthritis: a systematic review of cohort studies with focus on dose. Semin Arthritis Rheum 2013; 43: 144-151.

42 Prevoo ML, van't Hof MA, Kuper HH, et al. Modified disease activity scores that include twenty-eight-joint counts. Development and validation in a prospective longitudinal study of patients with rheumatoid arthritis. Arthritis Rheum 1995; 38: 44-48.

43 Judson MA, Baughman RP, Costabel U, et al. Safety and efficacy of ustekinumab or golimumab in patients with chronic sarcoidosis. Eur Respir J 2014; 44: 1296-1307. 